\title{
Socially structured games and their applications
}

Citation for published version (APA):

Herings, P. J. J., van der Laan, G., \& Talman, A. J. J. (2003). Socially structured games and their applications. METEOR, Maastricht University School of Business and Economics. METEOR Research Memorandum No. 009 https://doi.org/10.26481/umamet.2003009

Document status and date:

Published: 01/01/2003

DOI:

10.26481/umamet.2003009

Document Version:

Publisher's PDF, also known as Version of record

\section{Please check the document version of this publication:}

- A submitted manuscript is the version of the article upon submission and before peer-review. There can be important differences between the submitted version and the official published version of record.

People interested in the research are advised to contact the author for the final version of the publication, or visit the DOI to the publisher's website.

- The final author version and the galley proof are versions of the publication after peer review.

- The final published version features the final layout of the paper including the volume, issue and page numbers.

Link to publication

\footnotetext{
General rights rights.

- You may freely distribute the URL identifying the publication in the public portal. please follow below link for the End User Agreement:

www.umlib.nl/taverne-license

Take down policy

If you believe that this document breaches copyright please contact us at:

repository@maastrichtuniversity.nl

providing details and we will investigate your claim.
}

Copyright and moral rights for the publications made accessible in the public portal are retained by the authors and/or other copyright owners and it is a condition of accessing publications that users recognise and abide by the legal requirements associated with these

- Users may download and print one copy of any publication from the public portal for the purpose of private study or research.

- You may not further distribute the material or use it for any profit-making activity or commercial gain

If the publication is distributed under the terms of Article $25 \mathrm{fa}$ of the Dutch Copyright Act, indicated by the "Taverne" license above, 


\title{
Socially Structured Games and Their Applications
}

\author{
P. Jean-Jacques Herings ${ }^{1} \quad$ Gerard van der Laan ${ }^{2}$ Dolf Talman ${ }^{3}$
}

February 27, 2003

${ }^{1}$ P.J.J. Herings, Department of Economics and METEOR, Universiteit Maastricht, P.O. Box 616, 6200 MD Maastricht, The Netherlands.

E-mail: P.Herings@algec.unimaas.nl Homepage: http://www.fdewb.unimaas.nl/algec/

${ }^{2}$ G. van der Laan, Department of Econometrics and Tinbergen Institute, Free University, De Boelelaan 1105, 1081 HV Amsterdam, The Netherlands, e-mail: glaan@econ.vu.nl

${ }^{3}$ A.J.J. Talman, Department of Econometrics \& Operations Research and CentER, Tilburg University, P.O. Box 90153, 5000 LE Tilburg, The Netherlands, e-mail: talman@uvt.nl 


\begin{abstract}
In this paper we generalize the concept of a non-transferable utility game by introducing the concept of a socially structured game. A socially structured game is given by a set of players, a possibly empty collection of internal organizations on any subset of players, for any internal organization a set of attainable payoffs and a function on the collection of all internal organizations measuring the power of every player within the internal organization. Any socially structured game induces a non-transferable utility game. In the derived nontransferable utility game, all information concerning the dependence of attainable payoffs on the internal organization gets lost. We show this information to be useful for studying non-emptiness and refinements of the core.

For a socially structured game we generalize the concept of $\pi$-balancedness to social stability and show that a socially stable game has a non-empty socially stable core. In order to derive this result, we formulate a new intersection theorem that generalizes the KKM-Shapley intersection theorem. The socially stable core is a subset of the core of the game. We give an example of a socially structured game that satisfies social stability, whose induced non-transferable utility game therefore has a non-empty core, but does not satisfy $\pi$-balanced for any choice of $\pi$.

The usefulness of the new concept is illustrated by some applications and examples. In particular we define a socially structured game, whose unique element of the socially stable core corresponds to the Cournot-Nash equilibrium of a Cournot duopoly. This places the paper in the Nash research program, looking for a unifying approach to cooperative and non-cooperative behavior in which each theory helps to justify and clarify the other.
\end{abstract}

Key words: Non-transferable utility game, Core, Balancedness, Nash program 


\section{Introduction}

Many economic situations can be modeled as a non-transferable utility game (NTU-game). In such a game a set of payoffs is assigned to any coalition of players, being a non-empty subset of the set of all players. A well-known solution concept for cooperative games is the core, consisting of all payoff vectors that can be attained by the grand coalition and cannot be improved upon by any coalition. In Bondareva [5] and Shapley [20], the notion of balancedness has been introduced and it has been shown that a transferable utility game has a non-empty core if and only if the game is balanced. Scarf [19] has extended the balancedness condition to games with non-transferable utility and showed that every balanced NTU-game has a non-empty core. So, for NTU-games balancedness is sufficient but not necessary. Billera [4] has shown that an NTU-game has a non-empty core if it satisfies the condition of $\pi$-balancedness. Nevertheless, there exist cooperative games having a non-empty core and not being $\pi$-balanced for any $\pi$. Necessary and sufficient conditions for non-emptiness of the core have been given by Keiding and Thorlund-Petersen [11], and Predtetchinski and Herings [17]. The conditions in the latter paper constitute a natural generalization of $\pi$-balancedness, by allowing $\pi$ to vary continuously with the payoffs that a coalition can reach, and by allowing $\pi$ to be multiple-valued. The resulting condition is labeled $\Pi$-balancedness.

In this paper we generalize NTU-games and introduce the concept of a so-called socially structured game. An NTU-game summarizes the results of mutual cooperation by members of a coalition by a unique set of attainable payoffs. In many economic situations of interest, however, the players within a coalition may have several possibilities for choosing an internal organization. For instance, in order to run a firm, a group of workers has often multiple possibilities to organize itself, for instance according to different hierarchical structures with different numbers of levels and different span of controls. Within a chosen hierarchical structure the internal organization may differ in the distribution of the players over the positions within the hierarchy.

In general, the admissible internal organizations of a group of players depend on the specific application one has in mind. In running a firm it may be appropriate to choose the internal organization of a hierarchy, whereas in other situations communication structures or permutational structures, as studied for TU-games by Myerson [14] and Nowak and Radzik [16], respectively, might fit better, or ordered structures as studied in van der Laan, Talman and Yang [12] for NTU-games.

In the usual approach of an NTU-game internal organizations are taken into account by assigning to any coalition the set of payoff vectors that contains all payoff vectors attainable within some internal organization of that coalition. According to this approach all information about the payoff sets for the different internal organizations is neglected. 
The concept of a socially structured game exploits this information by allowing that a payoff set is assigned to any organizational structure within any coalition. The set of payoffs attainable to a group of players does not depend only on the set of players, but also on the internal organization of the group.

We further assume that to any possible internal organization of any coalition a power vector is associated, whose components reflect the relative strengths of the individual members of the coalition within the internal organization. Although we present a number of proposals made in the literature to determine the strength of an individual in a certain organizational structure, in this paper we will make no attempt to discuss the pros and cons of the various ways a power vector can be determined. Instead, we will treat the power vector as exogenously given, and consider its derivation as part of for instance the sociological literature. This treatment parallels the exogenous treatment of preferences in the economic literature.

We hold the point of view that the power of an individual within an internal organization of a coalition matters. If an individual in a certain internal organization has more power than another individual, then he is assumed to be able to increase his payoff at the expense of the other individual. This process can only be stopped by a credible threat of the other individual to leave the internal organization or to reorganize the current group of individuals. Such a threat is only credible if the deviating individual can guarantee his new coalition members at least the same payoff as before, and the individual has the power to enforce the outcome that leads to these payoffs.

An obvious question is whether this refined modeling of games can be used to define a new solution concept. In this paper we introduce the concept of the socially stable core. For a payoff vector to be in the socially stable core, there should be neither incentives to deviate from an economic point of view, nor from a social one. A payoff will be called economically stable if it is feasible for some admissible organization of the grand coalition and it is undominated, i.e. there is no subcoalition that can organize itself to give all its members a higher payoff. Socially motivated deviations do not occur when all individuals are equally powerful at the proposed payoff. This is formalized by considering the power vectors of all internal organizations that could realize the payoff. If there is a weighted sum of these power vectors that gives all individuals the same power, then individuals are said to be equally powerful at the proposed payoff. Obviously, the socially stable core is a subset of the core. In general, it is a proper subset of the core and therefore the socially stable core can be considered as a refinement of the core.

We define the property of social stability for a socially structured game and refer to games satisfying this property as socially stable games. It will be shown that a socially stable game has a non-empty socially stable core. To do so, we formulate an intersection 
theorem on the unit simplex that generalizes the well-known intersection theorem used by Shapley [20], see also Herings [8], Ichiishi [10], and van der Laan, Talman and Yang [13].

Socially stable games have a non-empty core. We show by an example that social stability of the socially structured game does not imply that there exists a $\pi$-system such that the induced coalitional game is $\pi$-balanced. From this we conclude that more information on the internal organization not only refines the core, but also may produce games for which non-emptiness of the core cannot be established by $\pi$-balancedness. Social stability leads therefore to conditions for the core to be non-empty that are in between $\pi$-balancedness and П-balancedness.

We demonstrate the usefulness of the concept of the socially stable core by an example resembling an unexpected relationship between non-cooperative and cooperative games. The non-cooperative Cournot quantity competition game between firms producing a homogeneous commodity will be reformulated as a socially structured game. The socially stable core of the game contains a unique element that corresponds to the Cournot-Nash equilibrium of the non-cooperative quantity competition game. We show also that for a non-cooperative leader-follower game, the Stackelberg equilibrium yields the unique element of the socially stable core of that game. For these models the cooperative concept of socially stable core is sustained by the non-cooperative Nash equilibrium outcome and reversely. From this viewpoint this paper contributes to the Nash research program, see for instance Nash [15], looking for a unifying theory in which each approach helps to justify and clarify the other. A final application concerns the case that each internal organization is represented by a graph whose nodes are the agents and whose edges reflect the dominance relations between agents in decision making. For this case we present a number of proposals in the literature concerning the modeling of the power vectors.

The structure of the paper is as follows. In Section 2 socially structured games are introduced as well as the associated solution concept of the socially stable core. In Section 3 a new intersection theorem is presented and it is proven that a socially stable game has a non-empty socially stable core. In Section 4 we provide an example of a socially stable game, whose induced NTU-game does not satisfy the $\pi$-balancedness condition for any choice of $\pi$. Section 5 contains examples and applications and Section 6 concludes.

\section{Socially Structured Games}

Aumann and Peleg [2] have introduced cooperative non-transferable utility games with a finite number of agents as games in which for each coalition of agents a certain subset of payoff vectors is available on which the coalition can agree. When a coalition agrees on a payoff vector in the attainable payoff set, then each player of the coalition receives a 
payoff as specified by this player's component of the payoff vector. A payoff vector that is attainable for the grand coalition lies in the core of the game if no coalition can improve upon this vector, i.e. if there does not exist a coalition and a vector in its payoff set that makes all players better off, see Aumann [1].

In this paper we generalize the concept of an NTU-game by allowing for the possibility that a coalition can organize itself in several ways, where every admissible internal organization of a coalition leads to a payoff set on which the members of the coalition can agree. The internal organization could be, for example, a structure where one agent is the leader of the coalition making all decisions, while all other agents of the coalition follow him, a structure in which all members of the coalition are in an equal position to each other and decisions are made by a unanimity or majority voting rule, or a hierarchy in which the agents are ordered on several levels. To any feasible internal organization corresponds a non-empty set of payoff vectors which the members of the coalition can guarantee themselves when this structure applies. We further assume that a power vector is associated to any admissible internal organization of any coalition. The components of this power vector reflect the relative strengths of the individual members of the coalition within the internal organization. For example, in a hierarchy the agent at the top of the hierarchy has more power within the coalition than the other coalition members, whereas within an internal organization in which the members are in an equal position to each other, all members have the same power. A socially structured game (SSG) is given by a set of admissible internal organizations and for any admissible internal organization a non-empty payoff set and a power vector.

More formally, we assume that there is a finite set of agents $N=\{1, \ldots, n\}$. Any coalition $S$, where $S$ is a non-empty subset of $N$, is able to choose one of a finite number of possible internal organizations. The number of feasible internal organizations for coalition $S$ is assumed to be finite and is denoted by $m_{S}$. We allow the number $m_{S}$ to be zero in which case there is no way the members of coalition $S$ are able to organize themselves and generate payoffs to all the members of the coalition. For singleton coalitions $S=\{i\}$, $i \in N$, we assume that $m_{\{i\}}=1$. The collection of feasible internal organizations of coalition $S$ is denoted by $\mathcal{I}^{S}$ and its elements are denoted by $I_{1}^{S}, \ldots, I_{m_{S}}^{S}$. The union over $S$ of all internal organizations for $S$ is denoted by $\mathcal{I}$.

The payoff sets associated with every internal organization of a coalition are represented by a mapping $v$ from $\mathcal{I}$ to the collection of non-empty subsets of $\mathbb{R}^{n}$. For every $S \subset N$ and $j=1, \ldots, m_{S}$, the set $v\left(I_{j}^{S}\right)$ is assumed to be cylindric with respect to $S$, where a non-empty set $X \subset \mathbb{R}^{n}$ is said to be cylindric with respect to $S$ if for any two vectors $x$ and $y$ in $\mathbb{R}^{n}$ with $x_{i}=y_{i}$ for all $i \in S$, it holds that $x \in X$ implies $y \in X$. When $x \in v\left(I_{j}^{S}\right)$ for some $I_{j}^{S} \in \mathcal{I}$, this means that if coalition $S$ organizes itself according to $I_{j}^{S}$ 
the members of the coalition can attain payoffs $\left(x_{i}\right)_{i \in S}$ for themselves, independent of what the agents outside $S$ are doing.

We further assume that to any possible internal organization of any coalition a power vector is associated, whose components reflect the relative strengths of the individual members of the coalition within the internal organization. We will present a number of proposals made in the literature to determine the power vector. For the purpose of this paper, the power vector is assumed to be exogenously given. The power of an agent within an internal organization is measured by a power vector function $p$ from $\mathcal{I}$ to $\mathbb{R}_{+}^{n} \backslash\left\{0^{n}\right\}$, where $\mathbb{R}_{+}^{n}$ is the nonnegative orthant of $\mathbb{R}^{n}$ and $0^{n}$ is the $n$-vector of zeros. For $I \in \mathcal{I}$ and $i=1, \ldots, n$, the number $p_{i}(I)$ denotes the power of agent $i$ within internal organization $I$. We assume that every player outside the coalition on which an internal organization is defined has zero power, i.e. for every $S \subset N$, and $j=1, \ldots, m_{S}$, we have that $p_{i}\left(I_{j}^{S}\right)=0$ for all $i \in N \backslash S$. Moreover, we assume that the power of every agent involved in the internal organization is nonnegative and that at least one of these agents has a positive power. For every $S \subset N$ and $j=1, \ldots, m_{S}$, it holds that $p_{i}\left(I_{j}^{S}\right) \geq 0$ for all $i \in S$ and $\sum_{i \in S} p_{i}\left(I_{j}^{S}\right)>0$. We now have the following definition of a socially structured game (SSG).

\section{Definition 2.1 (Socially Structured Game)}

$A$ socially structured game is given by the quadruple $\Gamma=(N, \mathcal{I}, v, p)$.

We are interested in payoff vectors that are socially and economically stable. If an individual in a certain organizational structure has more power than another individual, then he is assumed to be able to increase his payoff at the expense of the other individual. Such a payoff is not socially stable. This process can only be stopped by a credible threat of the other individual to leave the coalition or to reorganize the current coalition. Such a threat is only credible if the deviating individual can guarantee his coalition members at least the same payoff as before, and the individual has the power to enforce the outcome that leads to these payoffs. To define social stability of a payoff vector in an SSG $\Gamma=$ $(N, \mathcal{I}, v, p)$ more formally, we first define the power cone of a payoff vector. The power cone of a payoff $x$ is defined by

$$
P C(x)=\left\{y \in \mathbb{R}^{n} \mid y=\sum_{\{I \mid x \in v(I)\}} \lambda_{I} p(I), \lambda_{I} \geq 0 \text { for all } I\right\} .
$$

Notice that the power cone of an arbitrary payoff vector in $\mathbb{R}^{n}$ is indeed a, possibly empty, cone and is a subset of $\mathbb{R}_{+}^{n}$. The power cone of $x$ is equal to the set of all nonnegative linear combinations of power vectors of all internal organizations that are able to generate $x$. A payoff vector is called socially stable if the vector of ones is contained in its power cone. Let $e$ denote the $n$-dimensional vector of ones. 


\section{Definition 2.2 (Socially Stable Payoff)}

For a socially structured game $\Gamma=(N, \mathcal{I}, v, p)$ a payoff vector $x \in \mathbb{R}^{n}$ is socially stable if $P C(x)$ contains the vector $e$.

Social stability of a payoff vector $x$ means that nonnegative real numbers or weights can be assigned to the internal organizations that are able to generate $x$ in such a way that the weighted total power of every agent is equal to one and therefore the same for every agent. Sometimes it will be useful to define social stability of a collection of internal organizations without reference to a particular payoff vector.

\section{Definition 2.3 (Socially Stable Collection of Internal Organizations)}

A collection of internal organizations in $\mathcal{I},\left\{I_{1}, \ldots, I_{k}\right\}$, is socially stable if the system of equations $\sum_{j=1}^{k} \lambda_{j} p\left(I_{j}\right)=e$ has a nonnegative solution. A socially stable collection of internal organizations in $\mathcal{I}$ is minimal if no subset of it is socially stable.

A socially stable payoff vector is therefore a payoff vector whose components can be achieved by every element of a socially stable collection of internal organizations for its members.

A socially stable payoff vector may not be achieved by an internal organization on the grand coalition. In general, if a payoff vector $x$ can be sustained by an internal organization on the grand coalition we say that $x$ is feasible.

\section{Definition 2.4 (Feasible Payoff)}

For a socially structured game $\Gamma=(N, \mathcal{I}, v, p)$ a payoff vector $x \in \mathbb{R}^{n}$ is feasible if $x \in v(I)$ for some $I \in \mathcal{I}^{N}$.

Furthermore, social stability of a payoff vector does not imply that payoff improvements are impossible. A feasible payoff vector upon which improvements are impossible is called economically stable.

\section{Definition 2.5 (Economically Stable Payoff)}

For a socially structured game $\Gamma=(N, \mathcal{I}, v, p)$ a payoff vector $x$ is economically stable if $x$ is feasible and there does not exist an $\bar{I} \in \mathcal{I}^{S}$ for some $S \subset N$ and $y \in v(\bar{I})$ satisfying $y_{i}>x_{i}$ for all $i \in S$.

Economic stability of a payoff vector $x$ means that it is feasible and that there is no internal organization of a coalition that can make all members of that coalition better of than in $x$. Economic stability is a one-to-one translation of the concept of the core for NTU-games to SSG's. We will therefore also refer to the set of all economically stable payoffs of an SSG $\Gamma$ as the core of $\Gamma$.

The set of all socially and economically stable payoff vectors is called the socially stable core of the game. 


\section{Definition 2.6 (Socially Stable Core)}

The socially stable core of a socially structured game $\Gamma=(N, \mathcal{I}, v, p)$ consists of the set of socially and economically stable payoff vectors of $\Gamma$.

A payoff vector $x$ is an element of the socially stable core if there is an internal organization of the whole set of agents that is able to generate $x$ (feasibility), there is no internal organization on a coalition that is able to generate more payoff for its members (economic stability), and $x$ can be achieved by a socially stable collection of internal organizations (social stability).

\section{Non-emptiness of the Socially Stable Core}

In this section we give sufficient conditions for the non-emptiness of the socially stable core of a socially structured game. The most important condition is that the game itself is socially stable. A socially structured game is called socially stable if every socially stable payoff vector can be sustained by an internal organization on the grand coalition.

\section{Definition 3.1 (Socially Stable Game)}

A socially structured game $\Gamma=(N, \mathcal{I}, v, p)$ is socially stable if any socially stable payoff $x$ is feasible.

Besides social stability of the game the other conditions for non-emptiness of the socially stable core are rather standard and more technical. All payoff sets should be comprehensive, closed and bounded from above. Recall that every payoff set of an internal organization of a coalition $S$ is cylindric with respect to $S$ and that $m_{\{i\}}=1$ for all $i \in N$. In the sequel, the payoff set corresponding to the unique internal organization of the single player coalition $\{i\}, i \in N$, is denoted by $v(i)$ and the maximum payoff that agent $i$ can guarantee himself is given by the real number $\alpha_{i}$.

\section{Theorem 3.2 (Non-emptiness of the socially stable core)}

A socially structured game $\Gamma=(N, \mathcal{I}, v, p)$ has a non-empty socially stable core if

(i) for every $S \subset N$, for every $I \in \mathcal{I}^{S}$, the set $\left\{\left(x_{i}\right)_{i \in S} \mid x \in v(I)\right.$ and $x_{i} \geq \alpha_{i}$ for all $i \in$ $S\}$ is bounded,

(ii) for every $I \in \mathcal{I}$, the set $v(I)$ is closed and comprehensive,

(iii) the game is socially stable. 
Observe that (ii) together with the fact that $v(i)$ is cylindric with respect to $\{i\}$ for any $i \in N$ implies that $v(i)=\left\{x \in \mathbb{R}^{n} \mid x_{i} \leq \alpha_{i}\right\}$.

In order to prove the theorem we first give an intersection result on the $(n-1)$ dimensional unit simplex $\Delta$ defined by

$$
\Delta=\left\{q \in \mathbb{R}_{+}^{n} \mid \sum_{i=1}^{n} q_{i}=1\right\} .
$$

This intersection result is interesting in itself and generalizes the well-known KKM-Shapley intersection theorem (Shapley [20], see also Herings [8]).

\section{Lemma 3.3}

Let $\mathcal{I}$ be a finite collection of internal organizations, $p: \mathcal{I} \rightarrow \mathbb{R}_{+}^{n} \backslash\{0\}$ a power function, and let $\left\{C^{I} \mid I \in \mathcal{I}\right\}$ be a collection of closed subsets of $\Delta$ satisfying

$$
\cup_{I \in \mathcal{I}} C^{I}=\Delta \text {, }
$$

(ii) for every $q$ in the boundary of $\Delta$ it holds that $S \subset\left\{i \in N \mid q_{i}>0\right\}$ when $q \in C^{I}$ for some $I \in \mathcal{I}^{S}$.

Then there exists a socially stable collection $\left\{I_{1}, \ldots, I_{k}\right\}$ such that $\cap_{j=1}^{k} C^{I_{j}} \neq \emptyset$.

\section{Proof}

Without loss of generality we may normalize the power vector function such that $\sum_{i=1}^{N} p_{i}(I)=$ $n$ for every $I \in \mathcal{I}$. For $I \in \mathcal{I}$, let us define $c^{I}=e-p(I)$. Let the set $Y^{n}$ be defined by

$$
Y^{n}=\operatorname{conv}\left(\left\{c^{I} \mid I \in \mathcal{I}\right\}\right)
$$

where $\operatorname{conv}(X)$ denotes the convex hull of a set $X \subset \mathbb{R}^{n}$. Observe that $\sum_{j=1}^{n} c_{j}^{I}=0$ for all $I \in \mathcal{I}$ and hence $\sum_{j=1}^{n} y_{j}=0$ for all $y \in Y^{n}$. Next, define the correspondence $F: \Delta \rightarrow Y^{n}$ by

$$
F(q)=\operatorname{conv}\left(\left\{c^{I} \mid q \in C^{I}, I \in \mathcal{I}\right\}\right), q \in \Delta .
$$

Since the collection of subsets $\left\{C^{I} \mid I \in \mathcal{I}\right\}$ is a covering of $\Delta$, the set $F(q)$ is non-empty for all $q \in \Delta$. It is easily verified that, for every $q \in \Delta, F(q)$ is convex and compact and that $\cup_{q \in \Delta} F(q)$ is bounded. Moreover, since the sets $C^{I}, I \in \mathcal{I}$, are closed, the mapping $F: \Delta \rightarrow Y^{n}$ is an upper hemi-continuous mapping from the set $\Delta$ to the collection of subsets of the set $Y^{n}$. Further, both sets $\Delta$ and $Y^{n}$ are non-empty, convex, and compact. Next, let $H$ be the mapping from $Y^{n}$ to the collection of subsets of $\Delta$ defined by

$$
H(y)=\left\{\widehat{q} \in \Delta \mid q^{\top} y \leq \widehat{q}^{\top} y \text { for every } q \in \Delta\right\}, y \in Y^{n} .
$$

Clearly, for every $y \in Y^{n}$ the set $H(y)$ is non-empty, convex, and compact, and $H$ is upper hemi-continuous. Hence, the mapping $D$ from the non-empty, convex, compact set 
$\Delta \times Y^{n}$ into the collection of subsets of $\Delta \times Y^{n}$ defined by $D(q, y)=H(y) \times F(q)$ is upper hemi-continuous and for every $(q, y) \in \Delta \times Y^{n}$, the set $D(q, y)$ is non-empty, convex, and compact. According to Kakutani's fixed point theorem, the mapping $D$ has a fixed point on $\Delta \times Y^{n}$, i.e. there exist $q^{*} \in \Delta$ and $y^{*} \in Y^{n}$ satisfying $y^{*} \in F\left(q^{*}\right)$ and $q^{*} \in H\left(y^{*}\right)$.

Let $\alpha^{*}=q^{* \top} y^{*}$. From $q^{*} \in H\left(y^{*}\right)$ it follows that $q^{\top} y^{*} \leq \alpha^{*}$ for every $q \in \Delta$. By taking $q=e(i)$, where $e(i) \in \Delta$ denotes the $i$-th unit vector, we obtain that $y_{i}^{*} \leq \alpha^{*}$, $i=1, \ldots, n$. Hence,

$$
\left.\begin{array}{ll}
y_{i}^{*}=\alpha^{*} & \text { if } q_{i}^{*}>0 \\
y_{i}^{*} \leq \alpha^{*} & \text { if } q_{i}^{*}=0 .
\end{array}\right\}
$$

Since $\sum_{i=1}^{n} y_{i}^{*}=0$, we obtain also that $\alpha^{*} \geq 0$.

On the other hand, $y^{*} \in F\left(q^{*}\right)$ implies there exist nonnegative numbers $\lambda_{1}^{*}, \ldots, \lambda_{k}^{*}$ satisfying $\sum_{j=1}^{k} \lambda_{j}^{*}=1$ and $y^{*}=\sum_{j=1}^{k} \lambda_{j}^{*} c^{I_{j}}$ for a collection $\left\{I_{1}, \ldots, I_{k}\right\}$ of $k$ different internal organizations in $\mathcal{I}$ such that $q^{*} \in C^{I_{j}}$ for every $j, j=1, \ldots, k$. Without loss of generality we assume that $\lambda_{j}^{*}>0$ for every $j=1, \ldots, k$. Let $S_{j}$ be the set of agents on which $I_{j}$ is an internal organization, i.e. $I_{j} \in \mathcal{I}^{S_{j}}$ for $j=1, \ldots, k$. By condition (ii) we have that $q_{i}^{*}=0$ implies $i \notin S^{j}$ for every $j=1, \ldots, k$, and thus $c_{i}^{I_{j}}=1$. Hence,

$$
y_{i}^{*}=\sum_{j=1}^{k} \lambda_{j}^{*} c_{i}^{I_{j}}=1>0 \text { if } q_{i}^{*}=0 .
$$

Suppose there exists an index $i \in N$ such that $q_{i}^{*}=0$. Then it follows from the equations (1) and (2) that $y_{i}^{*}>0$ for all $i \in N$, which contradicts $\sum_{i=1}^{n} y_{i}^{*}=0$. Consequently, for all $i \in N$, we have that $q_{i}^{*}>0$ and thus $y_{i}^{*}=\alpha^{*}$. Together with $\sum_{i=1}^{n} y_{i}^{*}=0$ this proves that $y^{*}=0^{n}$. Hence

$$
\sum_{j=1}^{k} \lambda_{j}^{*} p\left(I_{j}\right)=e-\sum_{j=1}^{k} \lambda_{j}^{*} c^{I_{j}}=e-y^{*}=e
$$

and thus the collection $\left\{I_{1}, \ldots, I_{k}\right\}$ is socially stable. Since $q^{*} \in \cap_{j=1}^{k} C^{I_{j}}$, this completes the proof.

Q.E.D.

The proof of Theorem 3.2 follows by applying Lemma 3.3.

\section{Proof of Theorem 3.2}

Without loss of generality we assume that $0^{n} \in v(i)$ for every $i \in N$. To apply Lemma 3.3, we define a collection $\left\{C^{I} \mid I \in \mathcal{I}\right\}$ satisfying the conditions of the lemma and show that an intersection point of a collection of socially stable sets induces an element in the socially stable core of the game. For given $M>0$ and for any $q \in \Delta$, let the number $\lambda_{q}$ be given by

$$
\lambda_{q}=\max \left\{\lambda \in \mathbb{R} \mid-M q+\lambda e \in \cup_{I \in \mathcal{I}} v(I)\right\} .
$$


Since $0^{n} \in v(i)$ and because of conditions (i) and (ii) of the theorem, $\lambda_{q}$ exists and is positive for every $M>0$ and for any $q \in \Delta$. Moreover, following Shapley [20], using condition (i) of the theorem, the number $M>0$ can be chosen so large that for every $i \in N$ and $q \in \Delta, q_{i}=0$ implies that $i \notin S$ for any $S \subset N$ satisfying $-M q+\lambda_{q} e \in v(I)$ for some $I \in \mathcal{I}^{S}$. Now, for $I \in \mathcal{I}$, define

$$
C^{I}=\left\{q \in \Delta \mid-M q+\lambda_{q} e \in v(I)\right\}
$$

Since every $v(I), I \in \mathcal{I}$, is closed and comprehensive, the collection of sets $\left\{C^{I} \mid I \in \mathcal{I}\right\}$ is a family of closed sets covering $\Delta$ and satisfies also Condition (ii) of Lemma 3.3. Hence, there is a socially stable collection $\left\{I_{1}, \ldots, I_{k}\right\}$ of internal organizations in $\mathcal{I}$ such that $\cap_{j=1}^{k} C^{I_{j}} \neq \emptyset$. Let $q^{*}$ be a point in this intersection, so $q^{*} \in C^{I_{j}}$ for $j=1, \ldots, k$. Then the point $x^{*}=-M q^{*}+\lambda_{q^{*}} e$ lies in $\cap_{j=1}^{k} v\left(I_{j}\right)$, i.e. $x^{*}$ is a socially stable payoff vector supported by the socially stable collection $\left\{I_{1}, \ldots, I_{k}\right\}$. Since the game is socially stable we have that $x^{*} \in v\left(I^{*}\right)$ for some $I^{*} \in \mathcal{I}^{N}$, i.e. $x^{*}$ is feasible. To prove economic stability, suppose there exist an internal organization $I \in \mathcal{I}^{S}$ for some $S \subset N$ and a payoff vector $y \in v(I)$ such that $y_{i}>x_{i}^{*}$ for all $i \in S$. Since $v(I)$ is comprehensive and cylindric with respect to $S$, there is a $\mu>0$ such that $x^{*}+\mu e \in v(I)$. However, then $-M q^{*}+\left(\lambda_{q^{*}}+\mu\right) e \in v(I)$, which contradicts that $-M q^{*}+\lambda e \notin v(I)$ for any $\lambda>\lambda_{q^{*}}$. Hence, $x^{*}$ cannot be improved upon by any internal organization $I \in \mathcal{I}$, i.e. $x^{*}$ is also economically stable. This completes the proof.

Q.E.D.

Since the socially stable core of a socially structured game is a subset of the core of that game, we have the following corollary.

\section{Corollary 3.4}

Let $(N, \mathcal{I}, v, p)$ be an SSG satisfying the conditions of Theorem 3.2. Then the core of the game is non-empty.

We conclude this section with an example to show that the socially stable core of a socially structured game might be indeed a proper subset of the core.

Example 3.5 Let $(N, \mathcal{I}, v, p)$ be a socially structured game with $N=\{1,2\}$ and $m_{\{1,2\}}=$

2. The payoff set mapping $v$ is given by

$$
\begin{aligned}
& v(i)=\left\{x \in \mathbb{R}^{2} \mid x_{i} \leq 0\right\}, \quad i=1,2, \\
& v\left(I_{1}^{\{1,2\}}\right)=\left\{x \in \mathbb{R}^{2} \mid 2 x_{1}+x_{2} \leq 3\right\}, \\
& v\left(I_{2}^{\{1,2\}}\right)=\left\{x \in \mathbb{R}^{2} \mid x_{1}+2 x_{2} \leq 3\right\} .
\end{aligned}
$$


The power vector function $p$ is given by $p\left(I^{i}\right)=e(i)$, where $I^{i}$ denotes the unique internal organization on the singleton coalition $\{i\}, i=1,2, p_{1}\left(I_{1}^{\{1,2\}}\right)>p_{2}\left(I_{1}^{\{1,2\}}\right)$, and $p_{2}\left(I_{2}^{\{1,2\}}\right)>$ $p_{1}\left(I_{2}^{\{1,2\}}\right)$. The core of this game is the union of the two sets $\left\{x \in \mathbb{R}^{2} \mid 2 x_{1}+x_{2}=3,0 \leq\right.$ $\left.x_{1} \leq 1\right\}$ and $\left\{x \in \mathbb{R}^{2} \mid x_{1}+2 x_{2}=3,0 \leq x_{2} \leq 1\right\}$. The socially stable core contains only one element, the point $(1,1)$. All other points of the core are not socially stable. Notice that in this game there are four minimal socially stable collections of internal organizations, namely $\left\{I^{1}, I^{2}\right\},\left\{I_{1}^{\{1,2\}}, I^{2}\right\},\left\{I_{2}^{\{1,2\}}, I^{1}\right\}$, and $\left\{I_{1}^{\{1,2\}}, I_{2}^{\{1,2\}}\right\}$. Only the last collection sustains the unique element in the core that is socially stable.

\section{Social Stability and $\pi$-Balancedness}

In this section we consider the relationship between social stability of socially structured games and $\pi$-balancedness of coalitional games, as introduced in Billera [4]. To define $\pi$-balancedness, for any subset $S$ of the set $N=\{1, \ldots, n\}$, let $\pi^{S} \in \mathbb{R}_{+}^{n}$ be a power vector satisfying $\pi_{j}^{S}=0$ for $j \notin S$ and $\pi_{i}^{S}>0$ for $i \in S$. Then a collection $\left\{S_{1}, \ldots, S_{k}\right\}$ of subsets of $N$ is called $\pi$-balanced if there exists positive numbers $\lambda_{1}, \ldots, \lambda_{k}$, such that

$$
\pi^{N}=\sum_{j=1}^{k} \lambda_{j} \pi^{S_{j}} .
$$

Observe that for any $\pi$-system $\left\{\pi^{S} \mid S \subset N\right\}$, the collection containing only the grand coalition $N$ is balanced. Further, in case for all $S \subset N$ we take $\pi_{i}^{S}=1$ for all $i \in S$, $\pi$-balancedness reduces to the well-known balancedness as introduced by Shapley [20].

A coalitional game on $N$ is defined by a payoff set mapping $v^{c}: \mathcal{N} \rightarrow 2^{\mathrm{R}^{n}}$, where $\mathcal{N}=\{S \mid \emptyset \neq S \subset N\}$ is the collection of all non-empty subsets of $N$, assigning to any $S \in \mathcal{N}$ a non-empty payoff set $v^{c}(S) \subset \mathbb{R}^{n}$ which is cylindric with respect to $S$. For a given $\pi$-system, a coalitional game $\left(N, v^{c}\right)$ is $\pi$-balanced when for any $\pi$-balanced collection $\left\{S_{1}, \ldots, S_{k}\right\}$ it holds that

$$
\cap_{j=1}^{k} v^{c}\left(S_{j}\right) \subset v^{c}(N) .
$$

When any set $v^{c}(S)$ satisfies the conditions (i) and (ii) of Theorem 3.2, it is well-known that the coalitional game has a non-empty core when there exists a $\pi$-system for which the game is $\pi$-balanced. Clearly, any $\pi$-balanced coalitional game $\left(N, v^{c}\right)$ yields a socially stable game $(N, \mathcal{I}, v, p)$ with, for all $S \subset N, m_{S}=1, v\left(I_{1}^{S}\right)=v^{c}(S)$, and $p_{i}\left(I_{1}^{S}\right)=\pi_{i}^{S} / \pi_{i}^{N}$, $i \in N$. Since $\{N\}$ is $\pi$-balanced, $\{N\}$ is also socially stable and the socially stable core and the core coincide.

For a socially structured game $(N, \mathcal{I}, v, p)$ we may define the induced coalitional game $\left(N, v^{c}\right)$ by defining the payoff set mapping function $v^{c}$ on $\mathcal{N}$ by

$$
v^{c}(S)=\cup_{I \in \mathcal{I}^{S}} v(I), \emptyset \neq S \subset N,
$$


i.e. the induced NTU-game payoff set $v^{c}(S)$ of coalition $S \subset N$ is defined to be the union of all payoff sets assigned to the admissible internal organizations on the coalition $S$ of players. It is straightforward that the core of this induced coalitional game $\left(N, v^{c}\right)$ coincides with the core of the socially structured game $(N, \mathcal{I}, v, p)$.

In the previous section we have seen that the socially structure game has a nonempty socially stable core, and thus a non-empty core, when the game is socially stable. Hence, it follows immediately that the induced coalitional game has a non-empty core when the underlying socially structured game is socially stable. We now show that social stability of a socially structured game does not necessarily imply that the induced coalitional game satisfies $\pi$-balancedness for some $\pi$-system. The concept of a socially structured game may yield for no $\pi$-system $\pi$-balanced induced coalitional games, but whose core non-emptiness follows from the social stability of the original game. This is shown in the next example.

Example 4.1 Let $(N, \mathcal{I}, v, p)$ be a socially structured game with $N=\{1,2,3\}$ and $m_{\{1,2\}}=$ $2, m_{\{1,3\}}=m_{\{2,3\}}=0$ and $m_{S}=1$ for all other $S \subset N$. The payoff set mapping $v$ is given by

$$
\begin{aligned}
& v(i)=\left\{x \in \mathbb{R}^{3} \mid x_{i} \leq 0\right\}, \quad i=1,2,3, \\
& v\left(I_{1}^{\{1,2\}}\right)=\left\{x \in \mathbb{R}^{3} \mid 2 x_{1}+x_{2} \leq 3\right\} \\
& v\left(I_{2}^{\{1,2\}}\right)=\left\{x \in \mathbb{R}^{3} \mid x_{1}+2 x_{2} \leq 3\right\}
\end{aligned}
$$

and

$$
v\left(I^{N}\right)=v(3) \cap v\left(I_{1}^{\{1,2\}}\right) \cap v\left(I_{2}^{\{1,2\}}\right) .
$$

The power vector function $p$ is given by $p\left(I^{i}\right)=e(i)$, where $I^{i}$ denotes the unique internal organization on the singleton coalition $\{i\}, i=1,2,3, p\left(I_{1}^{\{1,2\}}\right)=(2,1,0)^{\top}, p\left(I_{2}^{\{1,2\}}\right)=$ $(1,2,0)^{\top}$ and $p\left(I^{N}\right)=(1,1,1)^{\top}$. This socially structured game is socially balanced. To show this, it should be observed that there are only five minimal socially stable collections, $\left\{I^{1}, I^{2}, I^{3}\right\},\left\{I_{1}^{\{1,2\}}, I_{2}^{\{1,2\}}, I^{3}\right\},\left\{I_{1}^{\{1,2\}}, I^{2}, I^{3}\right\}\left\{I_{2}^{\{1,2\}}, I^{1}, I^{3}\right\}$ and $\left\{I^{N}\right\}$. For each of these collections we have that the intersection of the payoff sets of the members of the collection is a subset of $v\left(I^{N}\right)$. For instance,

$$
v\left(I_{1}^{\{1,2\}}\right) \cap v(2) \cap v(3) \subset\left\{x \in \mathbb{R}^{3} \mid x_{1} \leq 1 \frac{1}{2}, x_{2} \leq 0, x_{3} \leq 0\right\} \subset v\left(I^{N}\right) .
$$

Because the game is socially stable, the socially stable core is non-empty. In fact, the payoff vector $(1,1,0)^{\top}$ is the unique element in the socially stable core and is also the unique core element. This payoff vector lies in $v\left(I^{N}\right)$ and no coalition can improve upon this outcome, so it is in the core. Further, there are no other core elements, since the 
agents 1 and 2 can improve upon each other point in $v\left(I^{N}\right)$ through one or both of their internal organizations $I_{1}^{\{1,2\}}$ and $I_{2}^{\{1,2\}}$. Finally, this payoff vector is sustained through the socially stable collection $\left\{I_{1}^{\{1,2\}}, I_{2}^{\{1,2\}}, I^{3}\right\}$.

We now consider the induced coalitional game. The payoff set mapping $v^{c}$ of this game is given by

$$
\begin{aligned}
& v^{c}(i)=v(i), \quad i=1,2,3, \\
& v^{c}(\{1,2\})=v\left(I_{1}^{\{1,2\}}\right) \cup v\left(I_{2}^{\{1,2\}}\right) \\
& v^{c}(N)=v\left(I^{N}\right)
\end{aligned}
$$

and $v^{c}(S)=\emptyset$ for all other $S$. Of course, again the payoff vector $(1,1,0)^{\top}$ is the unique element in the core of this coalitional game and thus the core is non-empty. However, there does not exist a $\pi$-system for which the game is $\pi$-balanced. To show this, first let $\left\{\pi^{S} \mid S \subset N\right\}$ be a $\pi$-system such that $\pi_{1}^{\{1,2\}}=\pi_{2}^{\{1,2\}}$. Then the collection $\{\{1,2\},\{3\}\}$ is $\pi$-balanced. However, $v^{c}(\{1,2\}) \cap v^{c}(3)$ is not contained in $v^{c}(N)$, for instance $x=\left(\frac{1}{2}, 2,0\right)^{\top}$ is in $v^{c}(\{1,2\}) \cap v^{c}(3)$ but not in $v^{c}(N)$. Hence, the game is not $\pi$-balanced for any $\pi$-system with $\pi_{1}^{\{1,2\}}=\pi_{2}^{\{1,2\}}$. Next, suppose this latter equality does not hold. In this case we assume without loss of generality that $\pi_{1}^{\{1,2\}}<\pi_{2}^{\{1,2\}}$. Then the collection $\{\{1,2\},\{1\},\{3\}\}$ is $\pi$-balanced. However, the payoff vector $x=(0,3,0)^{\top}$ is in $v^{c}(\{1,2\}) \cap v^{c}(1) \cap v^{c}(3)$ but not in $v^{c}(N)$ and again the game is not $\pi$-balanced. Hence, there does not exist a $\pi$-system for which the induced coalitional game is $\pi$-balanced, so that the non-emptiness of the core can not be concluded from the $\pi$-balancedness condition. This concludes the example.

We conclude this section by considering the socially stable core of a socially structured game as a subset of the core. For any element $x$ in the socially stable core of a game $\Gamma$, feasibility of $x$ implies that $x \in v(I)$ for some $I \in \mathcal{I}^{N}$. Moreover, there exists a socially stable collection $\mathcal{H} \subset \mathcal{I}$ sustaining $x$. We noticed already that that there does not need to be a socially stable collection of internal organizations on the whole set of agents. Therefore, $\mathcal{H}$ may contain internal organizations of proper subsets of $N$. Moreover, it might be that $x$ is sustained by several socially stable collections. Now, let $\mathcal{I}(x)$ be the 'supercollection' containing all internal organizations that can achieve $x$. This supercollection contains at least one internal organization of the grand coalition $N$ and typically some internal organizations on subsets of $N$. Economic stability implies that improvements are not possible and therefore $x$ cannot be in the interior of any of these payoff sets. So, we have the following corollary.

\section{Corollary 4.2 (Boundary property)}

For a payoff $x$ in the socially stable core of $\Gamma$, let $\mathcal{I}(x)$ be the collection of all internal organizations $I$ such that $x \in v(I)$. Then $x$ is on the boundary of $v(I)$ for all $I \in \mathcal{I}(x)$. 
The corollary says that the socially stable core typically selects payoff vectors in the core, which are on the boundary of several payoff sets. In general, an element $x$ of the socially stable core lies in the (relative) interior of the core if $\mathcal{I}(x)$ contains only internal organizations on $N$ and $x$ lies in the boundary of the core if $\mathcal{I}(x)$ contains at least one internal organization on a proper subset of agents.

\section{$5 \quad$ Applications and Examples}

In this section we consider some applications and examples of socially structured games and discuss conditions under which the socially stable core is non-empty and how this set differs from the core itself. First, we consider a duopoly with two profit maximizing firms. We model this economic setting as a socially structured game and show that under rather general conditions its socially stable core contains exactly one element. Depending on the set of admissible internal organizations, this unique socially stable core element corresponds to either the Cournot-Nash outcome of duopoly or the Stackelberg solution.

Next we consider socially structured games, in which every internal organization of a coalition is represented by a directed graph. From the literature several power vector functions for graphs are known and we will give sufficient conditions under which the socially stable core of a game in graph structure is non-empty. In case there is only (at most) one graph and corresponding payoff set for any coalition, we consider by means of some examples how the subset of the core selected by the socially stable core depends on the graph structure and the corresponding power vector function.

\subsection{Duopoly}

As a first application we consider an industry with two profit maximizing firms producing a homogeneous good. Each firm $i, i=1,2$, has a strictly convex, increasing and differentiable cost function $c^{i}\left(q_{i}\right)$, where $q_{i} \geq 0$ is the quantity produced by firm $i$, and $c^{i}(0)=0$. The demand $q$ for the good is given by the concave, decreasing and differentiable inverse demand function $p=P(q)$, saying that the total demand for the good equals the total production $q=q_{1}+q_{2}$ when the price $p$ is equal to $P(q)$. The profit function of both firms depends on the quantities produced by each of the firms and is for firm $i$ given by

$$
\pi^{i}\left(q_{i}, q_{j}\right)=q_{i} P\left(q_{i}+q_{j}\right)-c^{i}\left(q_{i}\right)
$$

A well-known solution concept for this duopoly model is the non-cooperative Cournot-Nash solution in which simultaneously each firm chooses an optimal quantity given the choice of 
the other firm. For given $i=1,2$, let $q_{j} \geq 0$ be the quantity chosen by firm $j \neq i$. Then the optimal reaction of firm $i$ is the quantity solving the profit maximization problem

$$
\max _{q_{i} \geq 0} q_{i} P\left(q_{i}+q_{j}\right)-c^{i}\left(q_{i}\right)
$$

Let $r^{i}\left(q_{j}\right)$ be the solution to this problem. Under the conditions stated above on the demand and cost functions, the reaction functions $r^{i}: \mathbb{R}_{+} \rightarrow \mathbb{R}_{+}, i=1,2$, are well-defined and continuous. A pair $\left(q_{1}^{N}, q_{2}^{N}\right)$ of quantities is called a Cournot-Nash equilibrium if

$$
q_{i}^{N}=r^{i}\left(q_{j}^{N}\right), \text { for } i, j=1,2, i \neq j
$$

We also consider the Stackelberg leader-follower quantity competition game. In this model one of the firms, called the follower, say firm $j$, responds with his optimal reaction $r^{j}\left(q_{i}\right)$ to the quantity $q_{i}$ set by the other firm, called the leader, firm $i, i \neq j$. So, firm $i$ 's profit when it chooses quantity $q_{i} \geq 0$ is given by

$$
\pi_{S}^{i}\left(q_{i}\right)=q_{i} P\left(q_{i}+r^{j}\left(q_{i}\right)\right)-c^{i}\left(q_{i}\right) .
$$

A pair of quantities $\left(q_{1}^{i}, q_{2}^{i}\right)$ is a Stackelberg solution of the non-cooperative market game with firm $i$ as leader and firm $j \neq i$ as follower when

(i) $\quad q_{j}^{i}=r^{j}\left(q_{i}^{i}\right)$,

(ii) $\quad q_{i}^{i}$ solves $\max _{q_{i} \geq 0} \pi_{S}^{i}\left(q_{i}\right)$.

Under the conditions on the demand and cost functions stated above, the profit maximization problem

$$
\max _{q \geq 0} q P(q)-c^{i}(q)
$$

of a monopolistic firm $i$ has a unique solution, say $q_{i}^{m}$. Let $M C^{i}: \mathbb{R}_{+} \rightarrow \mathbb{R}$ be given by $M C^{i}\left(q_{i}\right)=\frac{\partial c^{i}\left(q_{i}\right)}{\partial q_{i}}$, i.e. $M C^{i}(\cdot)$ is the marginal cost function of firm $i, i=1,2$. We now assume that also the following conditions hold.

(i) For both firms $i=1,2$, the Stackelberg leadership profit function $\pi_{S}^{i}$ is strictly concave in $q_{i}$.

(ii) For both firms $i=1,2$, it holds that $P\left(q_{i}^{m}\right)>M C^{j}(0), j \neq i$.

The first condition guarantees that there exists a unique Cournot-Nash equilibrium, see for instance Tirole [21], page 225-226. The second condition, saying that the market price in case firm $i$ operates as a monopolist is higher than the marginal cost of firm $j \neq i$ at $q_{j}=0$, guarantees that at the Cournot-Nash equilibrium both firms are on the market, i.e. $q_{i}^{N}>0$ for $i=1,2$. Observe that in a Cournot-Nash equilibrium each player has a 
nonnegative profit, because a firm always has the possibility to set its production equal to zero, making profit equal to zero. Furthermore, under the first condition also the profit maximizing problem of the leader in the Stackelberg game has a unique solution.

We show first that the Cournot-Nash model can be formulated as a socially structured NTU-game. There are two agents, so $N=\{1,2\}$. On the grand coalition of both players, we consider two internal organizations. For ease of notation, let $I_{i}, i=1,2$, denote the internal organization of $N$ in which player $i$ follows the decision of player $j \neq i$. Further, let $I^{i}$ denote the unique organization for player $i$ himself, $i=1,2$. Then the collection of feasible internal organizations is equal to $\mathcal{I}=\left\{I^{1}, I^{2}, I_{1}, I_{2}\right\}$. Next, we define the payoff sets $v(I)$ for $I \in \mathcal{I}$. The internal organization $I_{i}, i=1,2$, corresponds to the case where the firms play the Stackelberg game with player $j \neq i$ as the leader and firm $i$ as the follower. We assign to this internal organization the set of payoffs that can be attained when the other firm $j$ produces an arbitrarily chosen quantity $q_{j}$ and firm $i$ chooses his optimal reaction $q_{i}=r^{i}\left(q_{j}\right)$ to $q_{j}$, i.e.

$$
v\left(I_{i}\right)=\left\{x \in \mathbb{R}^{2} \mid \exists q_{j} \geq 0 \text { such that } x_{j} \leq \pi^{j}\left(q_{j}, r^{i}\left(q_{j}\right)\right), x_{i} \leq \pi^{i}\left(r^{i}\left(q_{j}\right), q_{j}\right)\right\}, i=1,2 .
$$

To define the sets $v\left(I^{j}\right), j=1,2$, we assume that firm $j$ is aware that the other player $i \neq j$ will never set a quantity above his optimal leadership quantity $q_{i}^{i}$ in the Stackelberg game. Each firm can get at least the (low) payoff to be realized as a follower in the Stackelberg game solution. This yields for the singleton coalitions the cylindrical payoff sets

$$
v(j)=v\left(I^{j}\right)=\left\{x \in \mathbb{R}^{2} \mid x_{j} \leq \pi^{j}\left(r^{j}\left(q_{i}^{i}\right), q_{j}^{i}\right)\right\}, j=1,2 .
$$

Since the profits are bounded from above by the monopoly profits and are closed and comprehensive by definition, the conditions (i) and (ii) of Theorem 3.2 are satisfied.

As power vector function we take $p\left(I^{i}\right)=e(i), i=1,2$. For $I_{1}$ and $I_{2}$ we assume that $p_{i}\left(I_{i}\right)<p_{j}\left(I_{i}\right), i=1,2, j \neq i$, in order to express that player $j$ as a leader has more power than player $i$ as a follower. From this power vector function it follows that there are only four minimal socially stable collections, $\left\{I^{1}, I^{2}\right\},\left\{I_{1}, I_{2}\right\}$ and $\left\{I^{i}, I_{i}\right\}$ for $i=1,2$. Since

$$
\begin{array}{ll}
v\left(I^{1}\right) \cap v\left(I^{2}\right)=\left\{x \in \mathbb{R}^{2} \mid x_{i} \leq \pi^{i}\left(r^{i}\left(q_{j}^{j}\right), q_{j}^{j}\right), i=1,2\right\} & \subset v\left(I_{1}\right) \cup v\left(I_{2}\right), \\
v\left(I_{1}\right) \cap v\left(I_{2}\right) & \subset v\left(I_{1}\right) \cup v\left(I_{2}\right), \\
v\left(I^{i}\right) \cap v\left(I_{i}\right)=\left\{x \in \mathbb{R}^{2} \mid x_{i} \leq \pi^{i}\left(r^{i}\left(q_{j}^{j}\right), q_{j}^{j}\right)\right\} \cap v\left(I_{i}\right) & \subset v\left(I_{1}\right) \cup v\left(I_{2}\right), i=1,2,
\end{array}
$$

it follows that any socially stable payoff vector $x$ is feasible for the grand coalition $N$. So, the game is socially stable and thus satisfies also condition (iii) of Theorem 3.2. Since all conditions of Theorem 3.2 are satisfied the game has a non-empty socially stable core.

Let $x^{*}=\left(x_{1}^{*}, x_{2}^{*}\right)^{\top}$ be a payoff vector in the socially stable core. Then there are three possibilities. First, suppose $x^{*}$ is supported by the socially stable collection $\left\{I^{1}, I^{2}\right\}$. 
Then, $x^{*} \in v\left(I^{i}\right)$ and thus $x_{i}^{*} \leq \pi^{i}\left(r^{i}\left(q_{j}^{j}\right), q_{j}^{j}\right), i=1$, 2, i.e. each firm gets a payoff at most equal to his profit he gets as follower in the Stackelberg game. This contradicts that $x^{*}$ is economically stable, since $\pi^{i}\left(r^{i}\left(q_{j}^{j}\right), q_{j}^{j}\right)<\pi^{i}\left(q_{i}^{N}, q_{j}^{N}\right)$ for $i=1,2$ and the payoff vector $\left(\pi^{1}\left(q_{1}^{N}, q_{2}^{N}\right), \pi^{2}\left(q_{2}^{N}, q_{1}^{N}\right)\right)^{\top}$ lies in $v\left(I_{1}\right)$ as well as in $v\left(I_{2}\right)$. Secondly, suppose $x^{*}$ is supported by $\left\{I^{i}, I_{i}\right\}$ for some $i=1,2$. Then $x^{*} \in v\left(I^{i}\right) \cap v\left(I_{i}\right)$. Because $x^{*} \in v\left(I_{i}\right)$ there exists a quantity $q_{j}^{*}$ such that

$$
x_{j}^{*} \leq \pi^{j}\left(q_{j}^{*}, r^{i}\left(q_{j}^{*}\right)\right) \text { and } x_{i}^{*} \leq \pi^{i}\left(r^{i}\left(q_{j}^{*}\right), q_{j}^{*}\right) .
$$

Because $x^{*} \in v\left(I^{i}\right)$ it follows that

$$
x_{i}^{*} \leq \pi^{i}\left(r^{i}\left(q_{j}^{j}\right), q_{j}^{j}\right)
$$

Since $x^{*}$ is economically stable, all three inequalities hold with equality. Hence,

$$
\pi^{i}\left(r^{i}\left(q_{j}^{*}\right), q_{j}^{*}\right)=\pi^{i}\left(r^{i}\left(q_{j}^{j}\right), q_{j}^{j}\right)
$$

implying that $q_{j}^{*}=q_{j}^{j}$ and the payoffs are the Stackelberg leader-follower game payoffs with player $j$ as leader and player $i$ as follower. However, then there exists a payoff vector $x$ in the set $v\left(I_{j}\right)$, with $j \neq i$ as the follower, that dominates $x^{*}$. To see this, observe that the profits of both players increase if player $i$ continues to play $r^{i}\left(q_{j}^{j}\right)$ but player $j$ decreases his quantity from $q_{j}^{j}$ to his optimal reaction $r^{j}\left(r^{i}\left(q_{j}^{j}\right)\right)$ against $r^{i}\left(q_{j}^{j}\right)$, yielding the payoff vector $x$ in $v\left(I_{j}\right)$ with $x_{i}=\pi^{i}\left(r^{i}\left(q_{j}^{j}\right), r^{j}\left(r^{i}\left(q_{j}^{j}\right)\right)\right)>x_{i}^{*}$ and $x_{j}=\pi^{j}\left(r^{j}\left(r^{i}\left(q_{j}^{j}\right)\right), r^{i}\left(q_{j}^{j}\right)\right)>x_{j}^{*}$. This contradicts that $x^{*}$ cannot be improved upon. Therefore, any socially stable core element is supported by the family $\left(I_{1}, I_{2}\right)$, i.e. $x^{*} \in v\left(I_{i}\right), i=1,2$. Because $x^{*} \in v\left(I_{2}\right)$ we have by definition of the set $v\left(I_{2}\right)$ that there exists a quantity $q_{1}^{*}$ such that

$$
x_{1}^{*} \leq \pi^{1}\left(q_{1}^{*}, r^{2}\left(q_{1}^{*}\right)\right) \text { and } x_{2}^{*} \leq \pi^{2}\left(r^{2}\left(q_{1}^{*}\right), q_{1}^{*}\right) .
$$

Analogously, from $x^{*} \in v\left(I_{1}\right)$ it follows that there exists a quantity $q_{2}^{*}$ such that

$$
x_{1}^{*} \leq \pi^{1}\left(r^{1}\left(q_{2}^{*}\right), q_{2}^{*}\right) \text { and } x_{2}^{*} \leq \pi^{2}\left(q_{2}^{*}, r^{1}\left(q_{2}^{*}\right)\right) .
$$

Since $x^{*}$ is economically stable we must have that all inequalities hold with equality and hence it follows that

$$
\pi^{1}\left(q_{1}^{*}, r^{2}\left(q_{1}^{*}\right)\right)=\pi^{1}\left(r^{1}\left(q_{2}^{*}\right), q_{2}^{*}\right) \text { and } \pi^{2}\left(q_{2}^{*}, r^{1}\left(q_{2}^{*}\right)\right)=\pi^{2}\left(r^{2}\left(q_{1}^{*}\right), q_{1}^{*}\right)
$$

From the uniqueness of the Nash equilibrium it follows that $q_{i}^{*}=r^{i}\left(q_{j}^{*}\right)$ and hence $q_{i}^{*}=q_{i}^{N}$, $i=1,2$. So, the quantities supporting the unique payoff vector in the socially stable core are the Cournot-Nash quantities. This gives the following result. 


\section{Result 5.1}

The socially stable core of the socially structured duopoly game $(N, \mathcal{I}, v, p)$ with $\mathcal{I}=$ $\left\{I^{1}, I^{2}, I_{1}, I_{2}\right\}$ contains a unique element $x^{*}$. The corresponding quantities $q_{1}^{*}$ and $q_{2}^{*}$ form the unique Cournot-Nash equilibrium for the non-cooperative quantity competition game.

The result shows that the Cournot-Nash outcome of the non-cooperative game is supported by the socially stable core outcome of an appropriately defined socially structured game. In fact, it should be noticed that the non-cooperative behavior is modeled by an appropriate choice of the collection of feasible internal organizations and corresponding payoff sets.

Next we consider the Stackelberg leader-follower quantity competition game. To formulate a socially structured game yielding as the unique socially stable core outcome the solution $\left(q_{1}^{j}, q_{2}^{j}\right)$ of the Stackelberg game with firm $j$ being the leader, we take as the collection of feasible internal organizations

$$
\mathcal{I}=\left\{I^{1}, I^{2}, I_{i}\right\}
$$

with $I^{1}, I^{2}$ and $I_{i}, i \neq j$, as defined above. We take the same payoff sets and power vectors as above for the three elements of $\mathcal{I}$, so that the game $(N, \mathcal{I}, v, p)$ satisfies the conditions (i) and (ii) of Theorem 3.2. Clearly, the only minimal socially stable collections are $\left\{I^{1}, I^{2}\right\}$ and $\left\{I_{i}, I^{i}\right\}$. It follows as above that for both collections the intersection of the payoff sets is in $v\left(I_{i}\right)$. Hence, the game is socially stable and thus also satisfies condition (iii) of Theorem 3.2. Therefore, the game has a non-empty socially stable core. By an analogously reasoning as in the Cournot-Nash case it follows that any payoff vector $x^{*}=\left(x_{1}^{*}, x_{2}^{*}\right)^{\top}$ in the socially stable core is supported by the socially stable collection $\left\{I_{i}, I^{i}\right\}$, so that $x^{*} \in v\left(I_{i}\right) \cap v\left(I^{i}\right)$ and the payoff vector $x^{*}$ is supported by the quantities $q_{j}^{*}=q_{j}^{j}$ and $q_{i}^{*}=r^{i}\left(q_{j}^{j}\right)=q_{i}^{j}$ for $i \neq j$. Notice that, in contrast to the Cournot-Nash case, these payoffs cannot be improved by a payoff vector in $v\left(I_{j}\right)$ by decreasing the quantity of the leader $j$ because now $I_{j}$ is not in the collection $\mathcal{I}$. So, the socially stable core contains a unique payoff vector, whose components are the Stackelberg leader-follower game payoffs with player $j$ as leader and player $i$ as follower. This gives the following result.

\section{Result 5.2}

The socially stable core of the socially structured game $\Gamma=(N, \mathcal{I}, v, p)$ with $\mathcal{I}=\left\{I^{1}, I^{2}, I_{i}\right\}$,

$i \in\{1,2\}$, contains a unique element $x^{*}$. The corresponding quantities $q_{1}^{*}$ and $q_{2}^{*}$ form the unique Stackelberg equilibrium for the non-cooperative quantity competition game with player $j$ being the leader and player $i$ being the follower.

\subsection{Games in Graph Structure}

A special class of socially structured games is what we would like to call games in graph structure, in which any admissible internal organization of a coalition is modeled by means 
of a (directed) graph. A graph $G$ is defined to be a pair $(V, A)$, where $V$ is a non-empty finite set of elements, called the vertices of the graph, and $A$ is a finite collection of ordered pairs of elements of $V$, called the arcs of the graph. In our setup $V$ is a subset $S$ of the grand coalition. Concerning $A$, two different agents $i, i^{\prime} \in S$ have no direct organizational relation in case both pairs $\left(i, i^{\prime}\right)$ and $\left(i^{\prime}, i\right)$ are not in $A, i$ weakly dominates $i^{\prime}$ if the pair $\left(i, i^{\prime}\right) \in A$, and $i$ dominates $i^{\prime}$ if the pair $\left(i, i^{\prime}\right) \in A$ and $\left(i^{\prime}, i\right) \notin A$. Although in principle we do not impose any restriction on a graph $G=(S, A)$, it is useful for the definition of the power vector function to exclude for all $i \in S$ the pairs $(i, i)$ from $A$.

Well-known examples of graph structures are the complete graph $A=\{(i, j) \mid i, j \in$ $S, i \neq j\}$, the empty graph $A=\emptyset$, a hierarchy (a graph that does not contain a directed sequence of edges from a node to itself), or a tree (a graph that contains for one specific node, the leader, a unique directed connected sequence of edges to any other node). If the coalition $S$ is able to generate payoff to its members when organized according to the graph $G=(A, S)$, we call $G$ feasible and denote the payoff set by $v(G)$. For any subset $S$ of $N$, the collection of all feasible graphs $G$ with vertex set $S$ is denoted by $\mathcal{G}^{S}$. The collection of all feasible graphs $\mathcal{G}$ is obtained by taking the union of $\mathcal{G}^{S}$ over all subsets $S$ of $N$. The payoffs are therefore determined by a mapping $v$ from $\mathcal{G}$ to the collection of non-empty subsets of $\mathbb{R}^{n}$ satisfying that for every graph $G \in \mathcal{G}^{S}$, the set $v(G) \subset \mathbb{R}^{n}$ is cylindric with respect to $S$.

To each feasible graph $G=(S, A)$ a power vector $p(G)$ is associated measuring the power of the nodes within $G$. Because any internal organization of a coalition $S$ is given by a graph on $S$, we can use one of the power vector functions for graphs known from the literature. To give some examples of proposed power vector functions, we define for $i \in S$ the sets of predecessors and successors of $i$ by

$$
P^{i}(G)=\{j \in S \mid(j, i) \in A\} \text { and } D^{i}(G)=\{j \in S \mid(i, j) \in A\},
$$

respectively, i.e. $P^{i}(G)$ is the set of all players by which $i$ is weakly dominated in $G$ and $D^{i}(G)$ is the set of all players in $G$ weakly dominated by $i$. A well-known method in graph theory to measure the power of a player in a graph is the score index, see for instance Behzad, Chartrand and Lesniak-Foster [3] or Rubinstein [18]. According to the score index, the power of a player $i \in S$ in the graph $G=(S, A)$ is equal to the number of elements in the set $D^{i}(G)$, i.e. the number of players in $S$ player $i$ is dominating. Another power index has been introduced by van den Brink [6], see also van den Brink and Gilles [7], according to which the power of a player $i$ in $G$ is given by $\sum_{j \in D^{i}(G)}\left|P^{j}(G)\right|^{-1}$. The interpretation of this dominance index is as follows. Initially, each player gets one point. This point is equally distributed amongst all his predecessors, so amongst all the players by which a player is weakly dominated. The power of a player is the sum of all his shares in the points of the players he weakly dominates. 
Finally, we give the so-called positional power index introduced in Herings, van der Laan and Talman [9]. While the score index of a player only depends on his number of successors and the dominance index of a player on the number of predecessors of each of his successors, the positional power of a player depends also on how powerful these successors are. More precisely, for a graph $G=(S, A)$ the positional power index is the solution to

$$
x_{i}=\sum_{j \in D^{i}(G)}\left(\frac{1}{n}+\frac{x_{j}}{n}\right), i \in S .
$$

It means that a player gets a power of $\frac{1}{n}$ for each weakly dominated player plus a fraction $\frac{1}{n}$ of the power of that player. The power index of a player is higher if he weakly dominates more powerful nodes. As shown in [9], the system (3) has a unique nonnegative nonzero solution. We now take some power index for the power vector function $p: \mathcal{G} \rightarrow \mathbb{R}^{n}$, i.e. for any graph $G \in \mathcal{G}$ on $S$, the powers $\left(p_{i}(G)\right)_{i \in S}$ are determined by a given power index for nodes in graphs, for instance the positional power index. This gives the following definition of a graph game.

\section{Definition 5.3 (Graph Game)}

A socially structured game in graph structure or graph game is given by the quadruple $\Gamma^{*}=(N, \mathcal{G}, v, p)$.

Social and economic stability are defined as before, but with respect to $\mathcal{G}$ instead of $\mathcal{I}$. From Theorem 3.2 it follows that a graph game $(N, \mathcal{G}, v, p)$ has a non-empty socially stable core if (i) the game is socially stable, (ii) for all $i \in N, G_{i}=(\{i\}, \emptyset) \in \mathcal{G}$ and $v\left(G_{i}\right)=$ $\left\{x \in \mathbb{R}^{n} \mid x_{i} \leq \alpha_{i}\right\}$ for some $\alpha_{i} \in \mathbb{R}$, and (iii) for all $S \subset N$, every set $v(G), G \in \mathcal{G}^{S}$, is comprehensive, closed and its individual rational points are bounded in $\mathbb{R}^{S}$.

As an example consider a firm that can be organized internally according to a tree in several ways. Not all tree structures might be allowed, for instance because of legal restrictions involved in the execution of certain tasks. Each feasible tree will generate possible payoffs for the people that are located on the nodes of the tree. Typically, these payoff sets are closed (continuity), comprehensive (free disposal) and bounded from above (finite profits). Suppose that, except for the singletons (outside options), only trees are feasible that involve all agents. Then it follows that either the profits corresponding to the outside options cannot be realized by any internal organization of the firm, in which case the firm cannot survive, or the socially stable core is non-empty. When the socially stable core is non-empty, there is a payoff vector, at least as good as the outside option vector, which cannot be improved upon by any other internal organization, and which is supported by a graph balanced collection of internal organizations. Any tree on the whole set of agents in this collection could be used to internally organize the firm. Notice that the 
balanced collection may contain one or more singletons, in which case the corresponding agents do not get more than their outside option.

As a second example we consider a firm with a fixed internal organization on $N$, being a hierarchy (tree) given by a linear ordering on the set of players, i.e. the agents can be numbered in such a way that the internal organization is given by the graph $(N, A)$ where $A=\{(i, i+1) \mid i=1, \ldots, n-1\}$. We further suppose that a coalition can only generate payoffs for themselves when they are connected in this graph $(N, A)$. So, for $S \subset N, \mathcal{G}^{S}$ contains at most one element. When $S=\{j, j+1, \ldots, k\}$ for some $j, k$ with $1 \leq j \leq k \leq n$, then $\mathcal{G}^{S}=\left\{\left(S, A^{S}\right)\right\}$ where $A^{S}=\{(h, h+1) \mid h=j, \ldots, k-1\}$ is the restriction of $A$ to $S$, otherwise $\mathcal{G}^{S}=\emptyset$. For ease of notation, in the following we denote a coalition of the form $\{j, j+1, \ldots, k\}$ by $[j, k]$. We further assume that for any graph $G=\left([j, k], A^{[j, k]}\right)$ the power index $p$ satisfies $p_{h}(G)>p_{h+1}(G), h=j, \ldots, k-1$. This is for instance the case when we take the positional power index. Since $\mathcal{G}$ only contains graphs of the form $\left([j, k], A^{[j, k]}\right)$, this implies that any socially stable collection of graphs must contain a graph of the form $\left([h, k], A^{[h, k]}\right)$ for any $h \in N$, i.e. any player $h$ must be in a coalition not containing players before $h$ in the hierarchy. So, any payoff vector $x$ in the socially stable core is sustained by a socially stable collection containing for any $h \in N$ a graph ([h, $\left.k], A^{[h, k]}\right)$, for some $k \geq h$. According to Corollary 4.2 it follows that when $x$ is a payoff vector in the socially stable core, for any $h \in N$ there is a coalition $[h, k]$ such that $x$ is on the boundary of the payoff set $v\left(\left([h, k], A^{[h, k]}\right)\right)$. Within this setting, the socially stable core selects a subset of the core such that at any payoff vector in this subset any player $h$ gets only a share in the payoff he can realize within some coalition $[h, k]$, i.e. a coalition containing some of its subsequent subordinates, but no superiors. So, within a firm with a linearly ordered hierarchy all profits that a player can realize in cooperation with superiors is distributed amongst its superiors. The example shows that by using information on the internal organization correctly, the framework of a socially structured game may provide more precise predictions about the outcome of economic situations.

Finally we consider an example of a graph game with a fixed internal organization on $N$, being the complete graph $(N, C)$, i.e. $C=\{(i, j) \mid i, j \in N, i \neq j\}$. Suppose that only the coalitions are able to generate payoffs by full cooperation, so for any $S \subset N$, $\mathcal{G}^{S}$ contains only the complete graph $\left(S, C^{S}\right)$. Within this framework we have a unique internal organization for every coalition. Since any graph is complete, we have that for every $G=\left(S, C^{S}\right)$ any power index $p$ discussed before satisfies $p_{i}(G)=p_{j}(G)$ for all $i, j \in S$. Moreover, in this case the collection consisting only of the complete graph on the grand coalition is socially stable. It follows that the socially stable core coincides with the core. 


\section{Concluding Remarks}

In this paper we have introduced socially structured games. The concept of socially structured games extends the standard NTU-game setting by taking into account the internal organization of a coalition and the associated power of players in this internal organization. We allow for several potential internal organizations of the same set of players, all potentially leading to different associated powers of the players involved.

We introduce the socially stable core as a refinement of the core. For a payoff to be in the socially stable core, it has to be both socially and economically stable. Social stability of a payoff is related to a condition of equal power of the players involved in internal organizations that are able to generate the payoff. Excess power of certain players will allow them to increase their payoff at the expense of others, an unstable situation. Economic stability reduces to the standard notion of the core. We have shown that the socially stable core of a socially structured game is non-empty if the game satisfies the condition of social stability, as well as some standard technical conditions. To prove this result, we formulate a new intersection theorem, which is of independent interest. Since the socially stable core refines the core, non-emptiness of the socially stable core implies non-emptiness of the core.

Any socially structured game induces an NTU-game. Although any balanced NTUgame induces a socially stable socially structured game, it is not necessarily the case that a socially stable socially structured game leads to a balanced NTU-game for some balancing weights. We have therefore shown that the additional structure of socially structured games can be used to provide new conditions that are sufficient for non-emptiness of the core.

We conclude the paper with two applications. The first application shows that the modeling of a duopoly with Cournot competition as a socially structured game leads to a socially stable core that coincides with the payoffs of the Cournot-Nash equilibrium. We also consider a special class of socially structured games in more detail, the so-called graph games. The literature provides a number of proposals to define the power of players, when their internal organization is modeled as a graph. We derive a number of first results for graph games. Since the class of graph games is sufficiently rich to encompass many economic phenomena of interest, we believe it to be a nice candidate for further research.

\section{References}

[1] R.J. Aumann, The core of a cooperative game without side payments, Transactions of the American Mathematical Society 98 (1961) 539-552. 
[2] R.J. Aumann and B. Peleg, Von Neumann-Morgenstern solutions to cooperative games without side payments, Bulletin of the American Mathematical Society 66 (1960) 173179.

[3] M. Behzad, G. Chartrand and L. Lesniak-Foster, Graphs and Digraphs, (Wadsworth, Belmont, 1979).

[4] L.J. Billera, Some theorems on the core of an $n$-person game without side payments, SIAM Journal on Applied Mathematics 18 (1970) 567-579.

[5] O.N. Bondareva, Some applications of linear programming methods to the theory of cooperative games, Problemy Kibernetiki 10 (1963) 119-139 (in Russian).

[6] R. van den Brink, Relational power in hierarchical organizations, Ph-D Dissertation, Tilburg University, The Netherlands, 1994.

[7] R. van den Brink and R.P. Gilles, Measuring domination in directed graphs, Social Networks 22 (2000) 1141-1157.

[8] P.J.J. Herings, An extremely simple proof of the K-K-M-S theorem, Economic Theory 10 (1997) 361-367.

[9] P.J.J. Herings, G. van der Laan and A.J.J. Talman, Measuring the power of nodes in digraphs, CentER Discussion paper 2001-72, Tilburg University, 2001.

[10] T. Ichiishi, Alternative version of Shapley's theorem on closed coverings of a simplex, Proceedings of the American Mathematical Society 104 (1988) 759-763.

[11] H. Keiding and L. Thorlund-Petersen, The core of a cooperative game without side payments, Journal of Optimization Theory and Applications 54 (1987) 273-288.

[12] G. van der Laan, A.J.J. Talman, and Z. Yang, Cooperative games in permutational structure, Economic Theory 11 (1998) 427-442.

[13] G. van der Laan, A.J.J. Talman and Z. Yang, Intersection theorems on polytopes, Mathematical Programming 84 (1999) 25-38.

[14] R.B. Myerson, Graphs and cooperation in games, Mathematics of Operations Research 2 (1977) 225-229.

[15] J. Nash, Two-person cooperative games, Econometrica 21 (1953) 128-140.

[16] A.S. Nowak and T. Radzik, The Shapley value for n-person games in generalized characteristic function form, Games and Economic Behavior 6 (1994) 150-161. 
[17] A. Predtetchinski and P.J.J. Herings, A necessary and sufficient condition for the non-emptiness of the core of a non-transferable utility game, METEOR Research Memorandum 02/19, Universiteit Maastricht, 2002.

[18] A. Rubinstein, Ranking the participants in a tournament, SIAM Journal of Applied Mathematics 38 (1980) 108-111.

[19] H. Scarf, The core of an $N$ person game, Econometrica 35 (1967) 50-69.

[20] L.S. Shapley, On balanced games without side payments, in: Mathematical Programming, eds. T.C. Hu and S.M. Robinson (Academic Press, New York, 1973) pp. 261-290.

[21] J. Tirole, The Theory of Industrial Organization, (MIT Press, Cambridge, 1988). 